\title{
Multivariable Analysis of Risk Factors for Enlargement of the Tracheoesophageal Puncture After Total Laryngectomy
}

\author{
Katherine A. Hutcheson, PhD ${ }^{1}$, Jan S. Lewin, $\mathrm{PhD}^{1}$, Erich M. Sturgis, M.D., MPH ${ }^{1,2}$, and Jan \\ Risser, $\mathrm{PhD}^{3}$ \\ ${ }^{1}$ Department of Head and Neck Surgery, The University of Texas M. D. Anderson Cancer Center \\ Houston, Texas 77030 USA \\ ${ }^{2}$ Department of Epidemiology, The University of Texas M. D. Anderson Cancer Center Houston, \\ Texas 77030 USA \\ ${ }^{3}$ Division of Epidemiology and Disease Control The University of Texas School of Public Health \\ Houston, TX 77030 USA
}

\begin{abstract}
Background-Enlarged tracheoesophageal puncture (TEP) is a challenging complication of surgical prosthetic voice restoration. Prevention of this complication requires identification of high risk individuals, as well as surgical and prosthetic correlates of TEP enlargement.

Methods-Multivariable logistic regression methods were used to analyze preoperative, perioperative, and postoperative risk factors for enlarged TEP in a 5-year retrospective cohort.

Results-Enlarged TEP only occurred in irradiated patients. Adjusting for length of follow-up and timing of TEP, advanced (N2 or N3) nodal disease ( $\mathrm{OR}_{\text {adjusted }}: 4.3,95 \%$ CI: 1.0-19.1), postoperative stricture ( $\mathrm{OR}_{\text {adjusted }}: 3.2,95 \% \mathrm{CI}$ : 1.2-8.6), and diagnosis of locoregional recurrence or distant metastasis after laryngectomy ( $\mathrm{OR}_{\text {adjusted }}$ 6.2, 95\% CI: 2.3-16.4) increased risk of enlarged TEP. Extended resection and preoperative nutritional status were also significantly associated with enlarged TEP. Prosthetic parameters did not significantly correlate with enlargement.
\end{abstract}

Conclusions-Development of enlarged TEP is a multifactorial process related to both baseline and postoperative factors.

\section{Keywords}

tracheoesophageal puncture; total laryngectomy; enlarged tracheoesophageal puncture; risk factors

\section{Introduction}

Tracheoesophageal puncture (TEP) has become the gold standard for alaryngeal voice rehabilitation after total laryngectomy since its introduction three decades ago. The current

Corresponding Author: Katherine A. Hutcheson, Ph.D. 1515 Holcombe Blvd, Unit 1445 Houston, TX 77030-4009 713-792-6513 karnold@mdanderson.org. 
method, introduced by Singer and Blom in 1980, ${ }^{(1)}$ relies on placement of a silicone voice prosthesis into a small, surgically created tracheoesophageal puncture, commonly referred to as the TEP. A one-way valved silicone voice prosthesis fitted within the TEP allows pulmonary air into the pharyngoesophagus for vibratory sound production when the tracheostoma is occluded. The prosthetic valve remains closed during swallowing to prevent aspiration. Enlargement of the TEP can be a serious complication that results in leakage of foods, liquids, or saliva around the prosthesis into the airway and may ultimately result in aspiration pneumonia. Although enlarged TEP and/or leakage around the voice prosthesis has been reported in up to $29 \%$ of TEP patients, ${ }^{(2)}$ the meta-analysis summary estimate from a recent systematic review suggests that the overall risk of enlarged puncture and/or leakage around the voice prosthesis is approximately $7 \% .^{(3)}$

Risk of enlarged TEP is likely elevated in certain subgroups of patients; however, few studies have addressed associated risk factors. There is conjecture that tissue changes associated with radiation damage, tumor recurrence, tobacco exposure, thyroid dysfunction, and malnutrition may predispose patients to enlarged puncture. ${ }^{(4-7)}$ While clinical experience supports these associations, epidemiological evidence remains sparse. ${ }^{(3)}$ Prosthetic variables, primarily diameter ${ }^{(4,8,9)}$ but also prosthetic insertion method, ${ }^{(9)}$ have been implicated as other potential risk factors for enlarged TEP. A systematic review, however, found insufficient evidence to conclude that use of a large diameter prosthesis increases risk of enlarged puncture and/or leakage around the prosthesis. ${ }^{(3)}$ A better understanding of risk factors for enlargement of the TEP is necessary to guide future research and evidence-based management of this complication. To date, a comprehensive analysis of risk factors for enlarged TEP has not been published. We sought to identify preoperative, perioperative, and postoperative risk factors for enlarged puncture using a retrospective cohort study design.

\section{Methods}

\section{Study Design and Inclusion/Exclusion Criteria}

A retrospective cohort study was conducted to identify risk factors for enlarged TEP. All patients who underwent TEP for voice restoration after total laryngectomy at The University of Texas MD Anderson Cancer Center (MDACC) between 05/2003 and 12/2008 were eligible for inclusion in the study. Patients whose surgical resection and/or TEP were performed at an outside institution and those with less than 3-months follow-up post-TEP were excluded. Two hundred thirty-seven eligible patients were identified by a search of the MDACC TEP Tracking Database. A total of 43 patients were excluded: 25 whose resection and/or TEP were performed at an outside facility, 17 with less than 3-months follow-up, and 1 whose voice prosthesis was placed in a persistent postoperative fistula rather than a surgically created TE fistula. Therefore, 194 patients were included in this analysis. Institutional review board approval and a waiver of informed consent were obtained.

\section{Institutional TEP Management}

Both primary and secondary TEP are performed at MD Anderson Cancer Center. The TEP is stented with a red rubber catheter (12-14-French) in the immediate postoperative setting. 
The initial voice prosthesis is typically placed 7-10 days postoperatively in conjunction with postoperative surgical clinic visits for primary TEPs and 3-5 days postoperatively for secondary TEPs. Standard practice is to dilate the TE tract to 18-French and insert a 16French non-indwelling prosthesis on the date of initial fitting. Patients are routinely followed for TEP management in the immediate postoperative period and then lifelong as needed.

Routine evaluation includes direct visualization of the TEP during liquid swallows to assess for leakage through and/or around the prosthesis, visual examination of the TEP, and measurement of TE tract length using commercially available tools (InHealth Technologies, Carpinteria, CA; Atos Medical, Hörby, Sweden). A variety of indwelling and nonindwelling prostheses are available for use within the institution in 16-, 17-, 20-, and 22.5French diameters (InHealth Technologies, Carpinteria, CA; Atos Medical, Hörby, Sweden). The speech pathologist selects and fits the voice prosthesis based on the unique presentation of each patient. Most patients try more than one type, brand, and diameter of prosthesis prior to ultimate determination of the optimal device.

\section{Data Collection}

Data sources included the MD Anderson TEP Tracking Database, the MD Anderson Tumor Registry, and review of the electronic medical record (EMR). Prosthetic variables and leakage patterns were captured prospectively in the MDACC TEP Tracking Database. Demographic data were captured using the MDACC Tumor Registry. EMR review was conducted by the primary author to collect and verify disease characteristics, medical comorbidities, and treatment variables.

The primary dependent variable was a diagnosis of enlarged TEP during the study period. For the purpose of this analysis, an enlarged TEP was defined as leakage around the voice prosthesis unresponsive to standard prosthetic management. It is routine practice to place a commercially-available voice prosthesis without an enlarged-flange after sizing the TE tract to determine the proper prosthetic length. Prostheses with an enlarged-flange are placed in the event of persistent leakage. Therefore, diagnosis of enlarged TEP included leakage around the prosthesis unresolved by replacement of a standard prosthesis or downsizing the length of the voice prosthesis, and/or leakage that required placement of a prosthesis with an enlarged flange. Leakage patterns were observed by direct visualization of the TEP during liquid swallows and were prospectively documented by the speech pathologist in the TEP Tracking Database and EMR. Diagnosis of enlarged TEP was confirmed by two authors (KAH, JSL).

Potential risk factors were collected for all laryngectomy subjects. Patient demographics, tobacco/alcohol history, medical comorbidities, disease characteristics, surgical variables, radiation and chemotherapy history, and prosthetic factors were recorded. Patient demographics included sex, age at time of laryngectomy, self-reported race/ethnicity at time of registration, marital status at time of registration, and institutional pay-code at time of registration. Institutional pay-codes were determined based on Federal Poverty Guidelines calculated by pre-tax gross annual family income and number of persons in household. Selfreported tobacco and alcohol exposure were coded per institutional classifications in the patient history database. ${ }^{(10,11)}$ Smoking status at time of laryngectomy was coded "never" 
( $<100$ lifetime cigarettes), "former" (quit at least 1 year preoperatively), or "current" (smoking within 1 year of surgery). Pack-year history of smoking was calculated. Selfreported alcohol exposure was coded "never" (never or "rarely" drink), "former" (at least 1 drink/month, but quit at least 1 year preoperatively), or "current" (currently drink at least 1 drink/month). Preoperative medical comorbidities included a physician diagnosis of diabetes recorded at preoperative assessment, $\mathrm{ACE}-27$ comorbidity score, ${ }^{(12)}$ body mass index (BMI), ${ }^{(13)}$ thyroid-stimulating hormone levels (normal range: $0.5-5.5 \mathrm{mcU} / \mathrm{mL}$ ), and serum albumin (normal range: $3.5-4.7 \mathrm{~g} / \mathrm{dL}$ ). The MD Anderson Cancer Center Clinical Chemistry Normal Values Bulletin was followed to define normal ranges. ${ }^{(14)}$ Levels of serum albumin depletion were classified as mild (2.8-3.5 g/dL), moderate (2.1-2.7 g/dL), or severe $(<2.1){ }^{(15)}$ Postoperative medical comorbidities included weight loss in the first postoperative month, stricture confirmed by endoscopic or fluoroscopic examination, and head and neck lymphedema. Postoperative weight loss was classified as significant (3-5\% in 1 month) or severe (>5\% in 1 month) according to institutional guidelines. ${ }^{(16)}$ Disease variables collected included tumor stage (classified by TNM criteria), tumor site, pathologic diagnosis, as well as diagnosis of locoregional recurrence, distant metastatic carcinoma, or new primary cancer after total laryngectomy. Perioperative variables included extent of surgery, type of reconstruction, and timing of TEP (primary or secondary procedure). Extent of surgery included receipt of partial or total pharyngectomy, neck dissection, subglottic/ tracheal resection, and/or thyroidectomy at the time of laryngectomy. Reconstruction was coded as "patch flap", including pedicled pectoralis flaps, radial forearm free flaps, and anterolateral thigh free flaps; or "circumferential flap", including tubed radial forearm free flaps, tubed ulnar free flaps, and tubed free anterolateral thigh flaps. No jejunal interpositions were used for pharyngeal reconstruction during the study period. In patients who underwent more than one TEP procedure $(n=13)$, the final TEP date, prior to TEP enlargement, was coded for the analysis. Prosthetic variables included the largest diameter of voice prosthesis worn by each patient at any point in time during the review period up until the time of enlargement (16-French, 17-French, 20-French, or 22.5-French), most common insertion method (retrograde using insertion stick or expandable insertion tube), and time to dilating the TE tract $\geq 20$-French (date first dilated minus date of TEP).

\section{Statistical Methods}

We sought to evaluate risk factors for enlarged TEP using unconditional multivariable logistic regression methods described by Hosmer and Lemeshow. ${ }^{(17)}$ Preoperative, perioperative, and postoperative risk factors were considered. Potential risk factors were thoroughly examined in univariable analyses prior to building the regression model. Unadjusted relative risk (RR) ratios and 95\% confidence intervals were calculated. Univariable logistic regression models containing each covariate were fit. Covariates of known clinical significance or with p-value $<0.25$ (likelihood ratio test chi-square) in the univariable logistic models were selected as candidates for the multivariable model. Length of follow-up and timing of TEP (primary versus secondary) were retained in all multivariable logistic regression models due to clinical relevance. An a priori threshold (zscore test, $\mathrm{p}<0.10$ ) was used to select variables for entry or removal during model building. Adjusted odds-ratios and 95\% confidence intervals were calculated based on the final multivariable logistic regression model. Odds-ratios were considered an unbiased estimate 
of relative risk in this cohort study. ${ }^{(18)}$ Postestimation statistics were calculated. Statistical significance was considered a-level 0.05 .

\section{Results}

\section{Patient Characteristics}

One hundred ninety-four patients met inclusion criteria for this analysis. The mean age was 62 years (standard deviation [sd]: 11, range: 37 - 86) and 84\% were male. Eighty-eight percent of patients were smokers and almost half of patients $(93 / 194,47.9 \%)$ were current drinkers. A majority of patients were white $(151 / 194,77.8 \%)$.

\section{Disease Characteristics}

Most patients $(177 / 194,91.2 \%)$ had a pathologic diagnosis of squamous cell carcinoma. Other diagnoses included thyroid (9/194, 4.6\%), sarcomatoid (4/194, 2.1\%), chondrosarcoma $(3 / 194,1.6 \%)$, and adenoidcystic $(1 / 194,0.5 \%)$ cancers. A majority $(103 / 194,53.1 \%)$ of patients underwent salvage laryngectomy after radiation or chemoradiation failure. Fifteen patients $(7.7 \%)$ underwent laryngectomy due to laryngeal dysfunction that included chronic aspiration and radionecrosis. Glottic $(87 / 194,44.9 \%)$ and supraglottic $(56 / 194,28.9 \%)$ primary tumors were most common. Other primary tumors included hypopharyngeal $(21 / 194,10.8 \%)$, subglottic $(15 / 194,7.7 \%)$, thyroid $(9 / 194,4.6 \%)$, and oropharyngeal $(4 / 194,2.1 \%)$; two additional patients $(1.0 \%)$ had a history of unknown primary head and neck cancers and underwent laryngectomy due to laryngeal dysfunction. Postoperative stricture was documented in $16.5 \%$ of patients (32/194) on average 12 months (sd: 12, range: 0-55) postoperatively. Additional comorbidities are summarized in Table 1.

\section{Treatment Characteristics}

Ninety-one percent of patients (177/194) received radiotherapy (median dose: 64 Gy, range: $10-80 \mathrm{~Gy})$. Eleven patients $(5.7 \%)$ had a history of more than one course of head and neck radiation. Only 17 patients had no head and neck radiation exposure. Forty-five percent of patients $(87 / 194)$ received chemotherapy.

Total laryngectomy without pharyngectomy was performed in 147 patients $(75.8 \%)$. Resection included a partial pharyngectomy in 26 patients $(13.4 \%)$ and total laryngopharyngectomy in $21(10.8 \%)$. Pharyngeal reconstruction was required in 50 patients, four of whom required delayed flap reconstruction for postoperative fistula repair. Primary TEP was performed in 119 patients (61.3\%) and secondary TEP in 75 (38.7\%).

Thirty percent developed locoregional recurrence $(30 / 194,15.5 \%)$, distant metastatic disease $(15 / 194,7.7 \%)$, or a new primary cancer $(13 / 194,6.7 \%)$ after laryngectomy; $64.4 \%$ $(125 / 194)$ were disease-free at last follow-up and 5.2\% (10/194) died of other causes. Mean length of follow-up was 24 months (sd: 17, range: 3-72) after TEP, and $90 \%$ of patients were followed greater than 6 months (inter-quartile range: 10-35 months). 


\section{Prosthetic Factors}

A variety of prosthetic types and diameters were tried by most patients. The largest prosthetic diameter worn by most patients was 20-French (133/194, 68.6\%). Retrograde placement using an insertion stick was most common (170/194, 87.6\%). Prosthetic variables were not coded for two patients whose TEP enlarged prior to placement of the initial prosthesis, one was diagnosed with level II nodal recurrence within 6 weeks of surgery, and the other presented with an enlarged TEP wearing a 14-French catheter one day after secondary TEP was performed.

\section{Incidence of Enlarged Puncture}

Thirty-six patients developed an enlarged TEP during the study period with an incidence of 18.6\% (95\% CI: $13.0 \%-24.1 \%$ ). Enlarged TEP was first diagnosed a median of 318 days after TEP (range: one to 1,653 days). Overall, 36\% (13/36) were diagnosed within six months of TEP, 22\% (8/36) between six and 12 months post-TEP, and 42\% (15/36) more than one year post-TEP. All enlarged TEPs were diagnosed after radiotherapy, a median of 19.2 months after the end date of radiotherapy (range: two days to 16.2 years).

\section{Risk Factors: Univariable Results}

Preoperative Patient Characteristics-Few demographic or preoperative medical variables were significantly associated with enlarged TEP in univariable analyses (Table 1). Specifically, sex, age, race and ethnicity, marital status, and institutional pay-code at registration were not significantly associated with enlarged TEP. A non-significant elevated risk of enlarged TEP was observed for females (RR 1.4, 95\% CI: 0.7-2.9). Smoking history at the time of laryngectomy was not significantly associated with enlarged puncture, however, enlarged TEP patients were 2.3 times more likely to be former smokers and 2.5 times more likely to be current smokers than never smokers.

Preoperative ACE-27 comorbidity score, diabetes, and thyroid-stimulating hormone were not statistically significantly associated with enlarged TEP. Non-significant elevated relative risks were observed for preoperative nutritional deficiencies, including mild (RR: 1.6, 95\% CI: 0.6-4.5) and moderate (RR: 2.2, 95\% CI: 0.8-5.8) serum albumin depletion and being underweight as defined by BMI (RR: 1.6, 95\% CI: 0.6-3.9). Interestingly, a significant dose response trend $(p=0.040)$ was observed for BMI categories, with substantially lower risk of enlarged TEP in obese and overweight patients, and higher risk in underweight patients.

Postoperative Patient Characteristics-Postoperative medical variables considered in univariable analysis included stricture, lymphedema, and weight loss in the first postoperative month (Table 1). A postoperative diagnosis of stricture significantly increased risk of enlarged TEP (RR: 2.2, 95\% CI: 1.2-4.1). Stricture was observed in 30.6\% of enlarged TEP cases compared with $13.3 \%$ of TEP controls without enlarged TEP. A nonsignificant elevated risk of enlarged TEP was observed for severe $(>5 \%)$ weight loss in the first postoperative month (RR: 1.7, 95\% CI: 0.9-3.4).

Disease Characteristics-A number of disease characteristics were significantly associated with enlarged TEP in univariable analyses (Table 2). Although enlarged TEP was 
not significantly associated with T-classification, staging of nodal disease was a significant predictor $(\mathrm{p}=0.012)$. In addition, risk of enlarged TEP was lowest in glottic/subglottic cancers and significantly highest in hypopharyngeal cancers (RR: 2.4, 95\% CI: 1.1-5.3); a non-significant elevated risk was observed in patients with supraglottic cancers (RR: 1.4, 95\% CI: 0.7-2.9).

Enlarged TEP was significantly more common in patients who were diagnosed with locoregional recurrence or distant metastatic cancer after laryngectomy compared with patients who remained disease-free (RR: 3.6, 95\% CI: 1.6-7.7). Among enlarged TEP patients, $8(22.2 \%)$ developed locoregional recurrence and 8 (22.2\%) developed distant metastasis after laryngectomy; only two of these had local peristomal recurrence near the TEP site. Incidence of enlarged puncture was $13.4 \%$ (9.5\% CI: 7.9\% - 19.0\%) in patients who remained disease-free after laryngectomy compared with $35.6 \%$ (95\% CI: $21.0 \%$ $50.1 \%$ ) in patients who developed locoregional recurrence or distant metastasis.

Surgical Variables-Few surgical variables were significantly associated with enlarged TEP in univariable analyses (Table 2). Risk of enlarged TEP was significantly higher after total laryngopharyngectomy compared with standard total laryngectomy (RR: 2.0, 95\% CI: 1.0-4.1). Additional surgical variables including neck dissection, thyroidectomy, and subglottic or tracheal resection were not significantly associated with enlarged TEP. Although enlarged TEP was 1.6 times more common in patients who received secondary TEP, this was not statistically significant (RR: 1.6, 95\% CI: 0.9-2.9).

Radiotherapy and Chemotherapy Variables-Univariable analyses of radiotherapy and chemotherapy data are shown in Table 2. Enlarged TEP was only diagnosed in irradiated patients $(\mathrm{p}=0.039)$, and all patients with enlarged TEP had completed radiotherapy prior to diagnosis of the complication. Relative risk ratios could not be estimated for many categorical radiotherapy variables using standard methods due to a zero count in the no radiation by enlarged TEP cell of the contingency table. Enlarged TEP was more common in patients who received postoperative radiotherapy compared to those who received definitive radiotherapy prior to salvage laryngectomy $(\mathrm{p}=0.029)$. Enlarged TEP patients were more likely to have had conventional 3D conformal radiotherapy fields than narrow-fields or IMRT, although this was not statistically significant $(\mathrm{p}=0.147)$. Total radiotherapy dose was significantly associated with enlarged TEP; there was a $16 \%$ increased risk of enlarged TEP for every $5 \mathrm{~Gy}$ increase in total radiation dose $(\mathrm{p}=0.012)$. Chemotherapy and enlarged puncture were significantly associated $(\mathrm{p}=0.033)$, with the highest risk observed for patients who received postoperative chemotherapy (RR: 2.2, 95\% CI: 1.2-4.0).

Prosthetic Variables-Prosthetic variables were not significantly associated with enlarged puncture in univariable analyses (Table 2). Risk of enlarged puncture was similar when stratified by the largest diameter of prosthesis worn, and relative risk ratios closely approximated the null for 20-French and 22.5-French diameter prostheses compared with 16- or 17-French diameter prostheses $(\mathrm{p}=0.979)$. Similar findings were observed comparing methods of prosthetic insertion ( $\mathrm{p}=0.584)$. 


\section{Multivariable Logistic Regression Model}

Based on univariable logistic regression results, 13 candidate variables were identified (LR test $\mathrm{p}<0.25$ ) for assessment in the multivariable logistic regression model (Tables 1 and 2). After adjusting for length of follow-up and timing of TEP, three variables met a priori criteria for inclusion in the final multivariable model (Table 3). Adjusting for length of follow-up and timing of TEP, advanced nodal disease (N2 or N3) increased risk of enlarged TEP 4-fold (OR adjusted: 4.3, 95\% CI: 1.0-19.1), postoperative stricture increased risk 3-fold

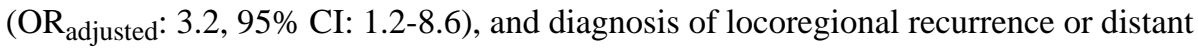
metastasis after laryngectomy increased risk of enlarged TEP 6-fold ( $\mathrm{OR}_{\text {adjusted }}$ 6.2, 95\% CI: 2.3-16.4). Postestimation testing indicated a well-fit model (Hosmer-Lemeshow goodness of fit, $\mathrm{p}=0.849$ ). Figure 1 illustrates the Kaplan-Meier estimates for enlarged TEP stratified by "high-risk" and "low-risk" patients. "High-risk" patients are those who had any of the 3 primary risk factors identified in the final multivariable logistic regression model including advanced nodal disease, postoperative stricture, and locoregional recurrence or distant metastasis after laryngectomy.

\section{Risk Factors for Enlarged TEP in Disease-Free Patients}

The incidence of enlarged puncture was $13.4 \%$ (95\% CI: 7.9\%-19.0\%) in the 149 patients who remained disease-free after laryngectomy compared with $35.6 \%$ (95\% CI: $21.0 \%-50.1 \%$ ) in the 45 patients who developed locoregional recurrence or distant metastasis after laryngectomy. As a secondary analysis, we sought to evaluate risk factors for enlarged TEP in patients who remained disease-free after laryngectomy. Univariable regression models restricted to disease-free patients indicated that radiation dose (OR for 5 Gy increase in total dose: 1.3, 95\% CI: 0.9-1.7), postoperative stricture (OR: 3.6, 95\% CI: 1.3-10.1), and length of follow-up (OR for 12-month follow-up: 1.9, 95\% CI: 1.4-2.7) were significantly associated with enlarged TEP $($ LRT, $\mathrm{p}<0.05)$ in patients who remained diseasefree after laryngectomy.

\section{Sensitivity Analysis using Cox Regression}

A sensitivity analysis was conducted using Cox regression methods to determine the effect of using time to event data on multivariable outcomes. The final multivariable Cox regression model identified the same risk factors as logistic regression methods. The effects of advanced nodal disease ( $\mathrm{HR}_{\text {adjusted }}: 3.2,95 \% \mathrm{CI}$ : 0.9-11.5), postoperative stricture

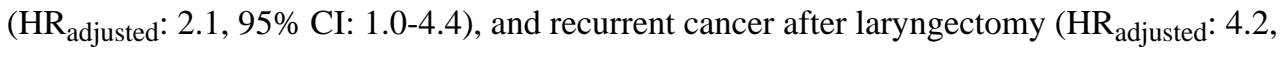
95\% CI: $2.0-8.9)$ were attenuated approximately $25 \%$ to $35 \%$ using Cox regression methods compared with logistic regression results.

\section{Discussion}

This study represents the first comprehensive assessment of preoperative, perioperative, and postoperative risk factors for enlarged TEP. Although a number of significant risk factors were identified in univariable analyses, three risk factors remained in the final multivariable model after adjusting for length of follow-up and timing of TEP; these included advanced (N2 or N3) N-stage, postoperative stricture, and diagnosis of locoregional recurrence or metastatic cancer after laryngectomy. Empirical and statistical evidence also supports an 
association between radiotherapy and enlarged TEP, despite our inability to include this factor in the multivariable analysis as enlarged TEP only occurred in irradiated patients. Other notable variables that may have a marked effect on the tendency of the TEP to enlarge are hypopharyngeal disease, total laryngopharyngectomy, and preoperative nutritional deficiencies, all of which were identified as potential risk factors in univariable analyses.

Advanced nodal disease stage (N2 or N3) elevated risk of enlarged TEP 4-fold, even after adjusting for other significant risk factors. In this study, postoperative radiotherapy was significantly more common in those with advanced nodal stage. Postoperative radiation also significantly increased risk of enlarged TEP in this cohort and it is, therefore, possible that nodal staging represented the effects of postsurgical radiation in the multivariable model. In addition, extended neck dissections required for patients with advanced nodal disease might have been associated with greater devascularization of the pharyngeal tissues perioperatively, predisposing to TEP enlargement. In any case, nodal staging is clearly known before surgery and may provide a simple preoperative indicator of risk for enlarged TEP.

Postoperative stricture was associated with a 3-fold increased risk of enlarged TEP in the adjusted multivariable model. Similar mechanisms of normal tissue toxicity may underlie postsurgical complications such as stricture and enlarged TEP. In addition, it is well documented that pharyngoesophageal pressure gradients during swallowing change after laryngectomy. ${ }^{(19-23)}$ Stricture further compounds these changes and contributes to heightened intraesophageal pressures which, when generated against an already compromised fistula, conceivably increase the risk of enlargement. Although most strictures are diagnosed postoperatively, early identification of stricture prior to TEP may help to prevent TEP enlargement. A careful interview will often elicit symptoms such as difficulty swallowing pills or solids foods as an indication of the need for radiographic or endoscopic evaluation to rule out stricture. When detected, treatment of stricture should be considered prior to secondary TEP.

The results of this study confirm the close association between recurrent cancer after laryngectomy and enlarged puncture. Although not the focus of their analyses, other authors have also acknowledged this potential relationship. ${ }^{(4,24)}$ The 6-fold higher risk of enlarged TEP in patients who developed locoregional recurrence or distant metastasis after laryngectomy was not explained by local effects of recurrence as only two patients developed recurrence in close proximity to the TEP site. Most recurrences were in the neck or lung. The pathophysiology of enlarged TEP in cases of remote recurrence remains unclear. Although the effects of systemic chemotherapeutic agents may play a role in enlargement, other factors must also be considered because enlarged TEP actually preceded the diagnosis of recurrence in nine patients. These patients exhibited symptoms of enlarged TEP prior to beginning systemic treatments for recurrent cancer. It is, therefore, possible that metabolic or systemic effects associated with the cancer itself may contribute to TEP enlargement. These findings reinforce the need to closely monitor for TEP-related complications in patients with recurrent disease after laryngectomy. Equally important, careful assessment of disease status is warranted in patients who exhibit an enlarged 
puncture, as these data suggest that retractable leakage around the prosthesis may be an early indicator of recurrent cancer in select cases.

In this cohort, more than $90 \%$ of patients received radiotherapy, which is known to cause inflammation and chronic fibrosis. ${ }^{(25,26)}$ Altough the time post-RT varied widely among enlarged TEP cases ( 2 days to 16.2 years), it was not surprising that enlarged TEP occurred exclusively in irradiated patients $(\mathrm{p}=0.039)$. The risk of enlarged TEP was highest in patients who received postoperative RT. This was unexpected, as current literature ${ }^{(7,27,28)}$ finds elevated complication rates in salvage laryngectomy cases in whom definitive radiotherapy doses are traditionally higher than postoperative doses. Our finding, however, may be better explained by the difference in fields targeted for definitive versus postoperative radiation delivery as opposed to differences in dose. Conventional postoperative radiotherapy delivers intermediate dose (54-57 Gy) to the entire surgical scar inclusive of the stoma and TEP site. In contrast, minimal esophageal dose is delivered in definitive fields. Effects of higher radiation doses delivered locally to the TEP in the postoperative setting may contribute to the higher incidence of enlarged TEP in these cases.

In addition to the three risk factors identified in the multivariable model, hypopharyngeal primary tumors, total laryngopharyngectomy, and preoperative nutritional deficiencies were associated with increased risk of enlarged TEP in univariable analyses. These results support those of other investigators who have found that extended laryngectomy is strongly associated with TEP complications. ${ }^{(27,29)}$ In our cohort, hypopharyngeal tumors and laryngopharyngectomy were significantly associated with enlarged TEP as well as the three significant risk factors identified in the multivariable model. Cross-tabulations indicated that postoperative stricture $(\mathrm{p}=0.003)$ and advanced $\mathrm{N}$-stage $(\mathrm{p}<0.001)$ were significantly more common in patients with hypopharyngeal disease, and recurrence after laryngectomy was significantly more common in patients who underwent laryngopharyngectomy $(\mathrm{p}=0.007)$. Although non-significant, preoperative serum album depletion and underweight BMI ratings also placed patients at increased risk of enlarged TEP. Nutritional deficiencies are known to negatively impact postoperative healing. ${ }^{(30)}$ It is also possible that patients with low BMI have a thin TE party wall, further predisposing to TEP enlargement; however, additional research is needed to clarify this relationship.

Our institutional practice of delaying TEP in patients perceived as high risk for postoperative complications due to factors such as severe radiation sequelae, malnutrition, or extended resection may have contributed to the higher incidence of enlarged TEP observed after secondary puncture. For instance, secondary TEP was significantly more common than primary TEP for patients in this cohort who underwent total laryngopharyngectomy $(\mathrm{p}<0.001)$ or salvage laryngectomy after definitive radiotherapy or chemoradiotherapy failure ( $\mathrm{p}=0.018)$. Similarly, the non-significant decreased risk of enlarged puncture observed when secondary TEP was performed in the outpatient clinic (OR: 0.6, 95\% CI: $0.2-1.8$ ) may have been a function of selection bias because more complex cases were performed in the operating room. As such, our results regarding timing (primary v. secondary) and type of procedure (operative v. TEP in clinic) should be interpreted with caution in the context of potentially confounding factors that contribute to these clinical decisions. 
Large diameter prostheses and expandable insertion tubes have been implicated as risk factors for enlarged puncture. ${ }^{(4,9)}$ This analysis, along with our previous systematic review, ${ }^{(3)}$ found no statistically significant associations between use of a large diameter prosthesis (20 or 22.5-French) and enlarged puncture. Beyond a lack of statistical significance, it was surprising that we observed no measurable differences in the incidence of enlarged puncture when stratified by prosthetic diameter and these relative risks closely approximated the null. Furthermore, these data are the first to statistically compare the effect of prosthetic insertion methods on TEP enlargement. Neither use of an expandable loading tube for prosthetic insertion nor large diameter prostheses were found to significantly increase risk of enlarged TEP. These data suggest that relative to oncologic and medical variables, prosthetic factors play little role in the risk of developing an enlarged puncture.

This study benefits from the close postoperative follow-up required for routine TEP management which, along with a prospective institutional TEP database and comprehensive EMR, strengthened our ability to obtain complete data despite a retrospective study design. Limitations, however, are inherent to retrospective observational studies. Length of followup varied among patients in this cohort, but all patients met a priori inclusion criteria of at least three months follow-up and the majority of the cohort was followed beyond two years. Furthermore, multivariable logistic regression models were adjusted for length of follow-up and similar results were obtained using Cox regression methods to account for time to event data. In addition, the influence of perioperative complications such as hematoma, blood loss, and pharyngocutaneous fistula was not analyzed in this study. Despite these limitations, our observational findings were enhanced by a large sample size, a clear case definition, inclusion of preoperative, perioperative, and postoperative risk factors, and the use of multivariable analysis methods.

\section{Conclusion}

A paramount goal of surgical prosthetic voice restoration is prevention of enlarged TEP which begins with identification of high risk patients. This multivariable analysis found three primary risk factors that may help to identify high risk patients including advanced Nstage, postoperative stricture, and locoregional recurrence or metastatic cancer after laryngectomy. Additional factors that are important to consider when assessing patients for TEP are radiotherapy, preoperative nutritional status, hypopharyngeal disease, and laryngopharyngectomy. These findings underscore the multifactorial nature of the problem and help to dispel suppositions that prosthetic diameter and insertion methods are key risk factors for enlarged puncture. Instead, the mere presence of a foreign body in a field compromised by medical and oncologic factors likely predisposes to enlargement of the fistula. Finally, preliminary evidence suggests that enlarged TEP may be an early sign of tumor recurrence in some cases, and heightened surveillance is indicated in the presence of this complication.

\section{References}

1. Singer MI, Blom ED. An endoscopic technique for restoration of voice after laryngectomy. Ann Otol Rhinol Laryngol. 1980; 89(6 Pt 1):529-33. [PubMed: 7458140] 
2. Gerwin JM, Culton GL, Gerwin JM, Culton GL. Prosthetic voice restoration with the tracheostomal valve: a clinical experience. Am J Otolaryngol. 1993; 14(6):432-9. [PubMed: 8285315]

3. Hutcheson KA, Lewin JS, Sturgis EM, Kapadia A, Risser J. Enlarged Tracheoesophageal Puncture After Total Laryngectomy: A Systematic Review and Meta-Analysis. Head Neck. [Epub ahead of press].

4. Acton LM, Ross DA, Sasaki CT, Leder SB. Investigation of tracheoesophageal voice prosthesis leakage patterns: patient's self-report versus clinician's confirmation. Head Neck. 2008; 30(5):61821. [PubMed: 18213715]

5. Hilgers FJ, Balm AJ. Long-term results of vocal rehabilitation after total laryngectomy with the lowresistance, indwelling Provox voice prosthesis system. Clin Otolaryngol Allied Sci. 1993; 18(6): 517-23. [PubMed: 8877233]

6. Jacobs K, Delaere PR, Vander Poorten VL. Submucosal purse-string suture as a treatment of leakage around the indwelling voice prosthesis. Head Neck. 2008; 30(4):485-91. [PubMed: 17979112]

7. Op de Coul BM, Hilgers FJ, Balm AJ, et al. A decade of postlaryngectomy vocal rehabilitation in 318 patients: a single Institution's experience with consistent application of provox indwelling voice prostheses. Arch Otolaryngol Head Neck Surg. 2000; 126(11):1320-8. [PubMed: 11074828]

8. Issing WJ, Fuchshuber S, Wehner M, Issing WJ, Fuchshuber S, Wehner M. Incidence of tracheooesophageal fistulas after primary voice rehabilitation with the Provox or the Eska-Herrmann voice prosthesis. Eur Arch Otorhinolaryngol. 2001; 258(5):240-2. [PubMed: 11548902]

9. Blom ED. Some comments on the escalation of tracheoesophageal voice prosthesis dimensions. Arch Otolaryngol Head Neck Surg. 2003; 129(4):500-2. [PubMed: 12707217]

10. Dube SR, Asman K, Malarcher A, Carabollo R. Cigarette smoking among adults and trends in smoking cessation -- United States, 2008. Morbidity and Mortality Weekly Report. 2009; 58(44): 1227-1232. [PubMed: 19910909]

11. Dawson, DA. Methodological issues in measuring alcohol use. National Institute on Alcohol Abuse and Alcoholism (NIAAA); Bethesda, MD: 2003.

12. Piccirillo JF. Importance of comorbidity in head and neck cancer. Laryngoscope. 2000; 110(4): 593-602. [PubMed: 10764003]

13. Defining Overweight and Obesity. Centers for Disease Control, Division of Nutrition, Physical Activity and Obesity, National Center for Chronic Disease Prevention and Health Promotion; Atlanta, GA: 2009.

14. Pathology \& Laboratory Medicine Bulletin of Information. The University of Texas M. D. Anderson Cancer Center; Houston, TX: 2010. Section of Clinical Chemistry Normal Values (Adult)..

15. Russell, MK.; McAdams, MP. Laboratory monitoring of nutritional status.. In: Matarese, LE.; Gottschlich, MM., editors. Contemporary Nutrition Support Practice: A Clinical Guide. W.B. Saunders Company; Philadelphia, PA: 1998. p. 47-63.

16. Nutrition Assessment of the Patient with Cancer. The University of Texas M. D. Anderson Cancer Center, Department of Clinical Nutrition; Houston, TX: Percent Weight Change..

17. Hosmer, DW.; Lemeshow, S. Applied logistic regression. 2nd ed.. John Wiley \& Sons, Inc.; Hoboken, NJ: 2000.

18. Szklo, M.; Nieto, FJ. Epidemiology: beyond the basics. 2nd ed.. Jones and Bartlett Publishers; Sudburry, MA: 2007.

19. McConnel FM, Hester TR, Mendelsohn MS, Logemann JA. Manofluorography of deglutition after total laryngopharyngectomy. Plast Reconstr Surg. 1988; 81(3):346-51. [PubMed: 3340668]

20. Dantas RO, Aguiar-Ricz LN, Gielow I, Filho FV, Mamede RC. Proximal esophageal contractions in laryngectomized patients. Dysphagia. 2005; 20(2):101-4. [PubMed: 16172817]

21. Choi EC, Hong WP, Kim CB, et al. Changes of esophageal motility after total laryngectomy. Otolaryngol Head Neck Surg. 2003; 128(5):691-9. [PubMed: 12748563]

22. Hanks JB, Fisher SR, Meyers WC, Christian KC, Postlethwait RW, Jones RS. Effect of total laryngectomy on esophageal motility. Ann Otol Rhinol Laryngol. 1981; 90(4 Pt 1):331-4. [PubMed: 7271143] 
23. Gates GA. Upper esophageal sphincter: pre and post-laryngectomy--a normative study. Laryngoscope. 1980; 90(3):454-64. [PubMed: 7359966]

24. Kress P, Schafer P, Schwerdtfeger FP, Kress P, Schafer P, Schwerdtfeger FP. [The custom-fit voice prosthesis, for treatment of periprothetic leakage after tracheoesophageal voice restoration]. Laryngorhinootologie. 2006; 85(7):496-500. [PubMed: 16586287]

25. Trotti A. Toxicity antagonists in head and neck cancer. Semin Radiat Oncol. 1998; 8(4):282-91. [PubMed: 9873106]

26. Wynn TA. Cellular and molecular mechanisms of fibrosis. J Pathol. 2008; 214(2):199-210. [PubMed: 18161745]

27. Starmer HM, Ishman SL, Flint PW, et al. Complications that affect postlaryngectomy voice restoration: primary surgery vs salvage surgery. Arch Otolaryngol Head Neck Surg. 2009; 135(11):1165-9. [PubMed: 19917932]

28. Emerick KS, Tomycz L, Bradford CR, et al. Primary versus secondary tracheoesophageal puncture in salvage total laryngectomy following chemoradiation. Otolaryngol Head Neck Surg. 2009; 140(3):386-90. [PubMed: 19248948]

29. Bozec A, Poissonnet G, Chamorey E, et al. Results of vocal rehabilitation using tracheoesophageal voice prosthesis after total laryngectomy and their predictive factors. Eur Arch Otorhinolaryngol. 267(5):751-8. [PubMed: 19890656]

30. Schwartz SR, Yueh B, Maynard C, Daley J, Henderson W, Khuri SF. Predictors of wound complications after laryngectomy: A study of over 2000 patients. Otolaryngol Head Neck Surg. 2004; 131(1):61-8. [PubMed: 15243559] 


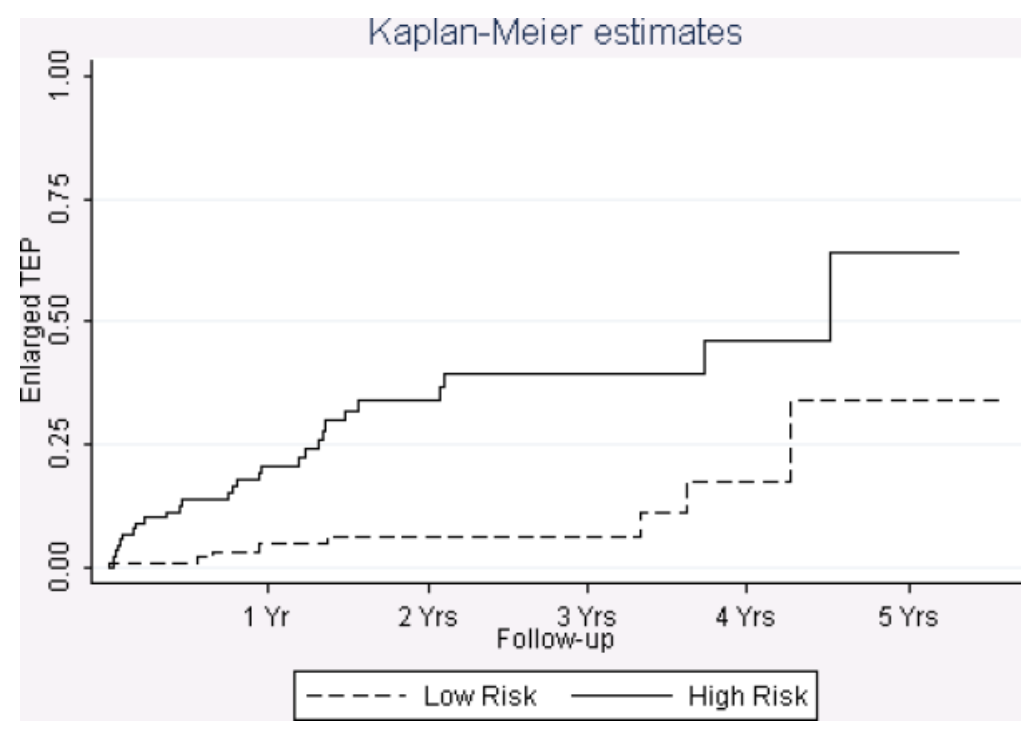

Figure 1. Risk-Stratified Kaplan-Meier Estimates for Enlarged TEP

Abbreviations: TEP, tracheoesophageal puncture; "high-risk", patients who had any of the 3 primary risk factors identified in the final multivariable logistic regression model (advanced nodal staging, postoperative stricture, or locoregional recurrence or distant metastasis after laryngectomy); "low risk", patients who had none of the 3 primary risk factors. 


\section{Table 1}

\section{Patient Characteristics}

\begin{tabular}{|c|c|c|c|c|}
\hline & Total No. (\%) & Enlarged No. (\%) & Crude RR (95\% CI) & LR-Test \\
\hline \multicolumn{5}{|l|}{ Sex } \\
\hline Male & $162(83.5 \%)$ & $28(17.3 \%)$ & 1.0 (referent) & \\
\hline Female & $32(16.5 \%)$ & $8(25.0 \%)$ & $1.4(0.7-2.9)$ & $\mathrm{p}=0.320$ \\
\hline \multicolumn{5}{|l|}{ Age } \\
\hline$<50$ years & $27(13.9 \%)$ & $4(14.8 \%)$ & 1.0 (referent) & \\
\hline $50-59$ years & $58(29.9 \%)$ & $13(22.4 \%)$ & $1.5(0.5-4.2)$ & \\
\hline $60-69$ years & $62(32.0 \%)$ & $11(17.7 \%)$ & $1.2(0.4-3.4)$ & \\
\hline$\geq 70$ years & $47(24.2 \%)$ & $8(17.0 \%)$ & $1.1(0.4-3.5)$ & $\mathrm{p}=0.823$ \\
\hline Mean yrs (sd) & $62.0(10.7 \%)$ & $61.1(10.7)$ & $1.0(1.0-1.0)$ & $\mathrm{p}=0.568$ \\
\hline \multicolumn{5}{|l|}{ Smoking Status ${ }^{*}$} \\
\hline Never & $24(12.4 \%)$ & $2(8.3 \%)$ & 1.0 (referent) & \\
\hline Former & $77(40.0 \%)$ & $15(19.5 \%)$ & $2.3(0.6-9.5)$ & \\
\hline Current & $93(47.9 \%)$ & $19(20.4 \%)$ & $2.5(0.6-9.8)$ & $\mathrm{p}=0.324$ \\
\hline \multicolumn{5}{|c|}{ Smoke after Radiotherapy, if salvage ${ }^{*}$} \\
\hline No & $76(39.2 \%)$ & $15(19.7 \%)$ & 1.0 (referent) & \\
\hline Yes & $32(16.5 \%)$ & $3(9.4 \%)$ & $0.5(0.1-1.5)$ & $\mathrm{p}=0.386$ \\
\hline N/A or Missing & $86(44.3 \%)$ & $1(16.7 \%)$ & -- & \\
\hline \multicolumn{5}{|l|}{ Alcohol ${ }^{*}$} \\
\hline Never & $47(24.2 \%)$ & $8(17.0 \%)$ & 1.0 (referent) & \\
\hline Former & $54(27.8 \%)$ & $11(20.4 \%)$ & $1.2(0.5-2.7)$ & \\
\hline Current & $93(47.9 \%)$ & $17(18.3 \%)$ & $1.1(0.5-2.3)$ & $\mathrm{p}=0.907$ \\
\hline \multicolumn{5}{|l|}{ Race/Ethnicity ${ }^{*}$} \\
\hline White & $151(77.8 \%)$ & $28(18.5 \%)$ & 1.0 (referent) & \\
\hline Black & $21(10.8 \%)$ & $4(19.1 \%)$ & $1.0(0.4-2.6)$ & \\
\hline Hispanic & $15(7.7 \%)$ & $3(20.0 \%)$ & $1.1(0.4-3.1)$ & \\
\hline Other & $7(3.6 \%)$ & $1(14.3 \%)$ & $0.8(0.1-4.9)$ & $\mathrm{p}=0.990$ \\
\hline \multicolumn{5}{|l|}{ Marital Status $^{*}$} \\
\hline Married & $136(70.1 \%)$ & $26(19.1 \%)$ & 1.0 (referent) & \\
\hline Not married & $58(29.9 \%)$ & $10(17.2 \%)$ & $0.9(0.5-1.7)$ & $\mathrm{p}=0.757$ \\
\hline \multicolumn{5}{|l|}{ Paycode at Registration ${ }^{*}$} \\
\hline Responsible $50 \%-100 \%$ bill & $173(89.2 \%)$ & $32(18.5 \%)$ & 1.0 (referent) & \\
\hline Responsible $0 \%$ bill & $21(10.8 \%)$ & $4(19.1 \%)$ & $1.0(0.4-2.6)$ & $\mathrm{p}=0.951$ \\
\hline \multicolumn{5}{|l|}{ Diabetes $^{*}$} \\
\hline No & $171(88.1 \%)$ & $34(19.9 \%)$ & 1.0 (referent) & \\
\hline Yes & $23(11.9 \%)$ & $2(8.7 \%)$ & $0.4(0.1-1.7)$ & $\mathrm{p}=0.161^{+}$ \\
\hline
\end{tabular}




\begin{tabular}{|c|c|c|c|c|}
\hline & Total No. (\%) & Enlarged No. (\%) & Crude RR $(95 \%$ CI) & LR-Test \\
\hline None/mild (0-1) & $129(66.5 \%)$ & $22(17.1 \%)$ & 1.0 (referent) & \\
\hline Moderate/severe (2-3) & $65(33.5 \%)$ & $12(21.5 \%)$ & $1.3(0.7-2.3)$ & $\mathrm{p}=0.452$ \\
\hline \multicolumn{5}{|l|}{ Albumin ${ }^{*}$} \\
\hline WNL & $136(70.1 \%)$ & $23(16.9 \%)$ & 1.0 (referent) & \\
\hline Mild depletion & $11(5.7 \%)$ & $3(27.3 \%)$ & $1.6(0.6-4.5)$ & \\
\hline Moderate depletion & $8(4.1 \%)$ & $3(37.5 \%)$ & $2.2(0.8-5.8)$ & $\mathrm{p}=0.314$ \\
\hline Missing & $39(20.1 \%)$ & $7(18.0 \%)$ & -- & \\
\hline \multicolumn{5}{|l|}{$\mathrm{BMI}^{*}$} \\
\hline Healthy & $76(39.2 \%)$ & $16(21.1 \%)$ & 1.0 (referent) & \\
\hline Underweight & $12(6.2 \%)$ & $4(33.3 \%)$ & $1.6(0.6-3.9)$ & \\
\hline Overweight & $69(35.6 \%)$ & $11(15.9 \%)$ & $0.8(0.4-1.5)$ & \\
\hline Obese & $35(18.0 \%)$ & $3(8.6 \%)$ & $0.4(0.1-1.3)$ & $\mathrm{p}=0.188^{\frac{t}{t}}$ \\
\hline Missing & $2(1.0 \%)$ & $2(100.0 \%)$ & -- & \\
\hline \multicolumn{5}{|l|}{$\mathrm{TSH}^{*}$} \\
\hline WNL & $126(65.0 \%)$ & $26(20.6 \%)$ & 1.0 (referent) & \\
\hline Low & $11(5.7 \%)$ & $2(18.2 \%)$ & $0.7(0.2-2.1)$ & \\
\hline Elevated & $21(10.8 \%)$ & $3(14.3 \%)$ & $0.9(0.2-3.2)$ & $\mathrm{p}=0.777$ \\
\hline Missing & $36(18.6 \%)$ & $5(13.9 \%)$ & -- & \\
\hline \multicolumn{5}{|c|}{ Weight Loss $1^{\text {st }}$ Month Post-TL ${ }^{\dagger}$} \\
\hline Not significant & $89(45.9 \%)$ & $17(19.1 \%)$ & 1.0 (referent) & \\
\hline Significant (3-5\%) & $28(14.4 \%)$ & $5(17.9 \%)$ & $0.9(0.4-2.3)$ & \\
\hline Severe $(>5 \%)$ & $30(15.5 \%)$ & $10(33.3 \%)$ & $1.7(0.9-3.4)$ & $\mathrm{p}=0.250$ \\
\hline Missing & $47(24.2 \%)$ & $4(8.5 \%)$ & -- & \\
\hline \multicolumn{5}{|l|}{ Stricture $^{\dagger}$} \\
\hline No & $162(83.5 \%)$ & $25(15.4 \%)$ & 1.0 (referent) & \\
\hline Yes & $32(16.5 \%)$ & $11(34.4 \%)$ & $2.2(1.2-4.1)$ & $\mathrm{p}=0.018^{\frac{t}{t}}$ \\
\hline \multicolumn{5}{|l|}{ Lymphedema $^{\dagger}$} \\
\hline No & $140(72.2 \%)$ & $25(17.9 \%)$ & 1.0 (referent) & \\
\hline Yes & $54(27.8 \%)$ & $11(20.4 \%)$ & $1.1(0.6-2.2)$ & $\mathrm{p}=0.689$ \\
\hline
\end{tabular}

Abbreviations: RR, relative risk; LR, likelihood ratio chi-square test; sd, standard deviation; WNL, within normal limits; BMI, body mass index; TL, total laryngectomy

* Preoperative variable

${ }^{\dagger}$ Postoperative variable

* Candidate variable for multivariable logistic regression model 
Table 2

Disease and Treatment Variables

\begin{tabular}{|c|c|c|c|c|}
\hline & Total No. (\%) & Enlarged TEP No. (\%) & Crude RR (95\% CI) & LR-Test \\
\hline \multicolumn{5}{|l|}{$\mathrm{T}$ classification } \\
\hline $\mathrm{T} 2$ & $9(4.6 \%)$ & $2(22.2 \%)$ & 1.0 (referent) & \\
\hline $\mathrm{T} 3$ & $19(9.8 \%)$ & $3(15.8 \%)$ & $0.7(0.1-3.5)$ & \\
\hline $\mathrm{T} 4$ & $57(29.4 \%)$ & $13(22.8 \%)$ & $1.0(0.3-3.8)$ & \\
\hline $\mathrm{TX}$ or missing & $5(2.6 \%)$ & $0(0.0 \%)$ & -- & \\
\hline Recurrent & $104(53.6 \%)$ & $18(17.3 \%)$ & $0.8(0.2-2.8)$ & $\mathrm{p}=0.825$ \\
\hline \multicolumn{5}{|l|}{$\mathrm{N}$ classification } \\
\hline No & $41(21.1 \%)$ & $3(7.3 \%)$ & 1.0 (referent) & \\
\hline N1 & $12(6.2 \%)$ & $3(25.0 \%)$ & $3.4(0.8-14.8)$ & \\
\hline $\mathrm{N} 2$ & $32(16.5 \%)$ & $12(37.5 \%)$ & $5.1(1.6-16.6)$ & \\
\hline $\mathrm{N} 3$ & $2(1.0 \%)$ & $0(0.0 \%)$ & -- & \\
\hline $\mathrm{NX}$ or missing & $3(1.5 \%)$ & $0(0.0 \%)$ & -- & \\
\hline Recurrent & $104(53.6 \%)$ & $18(17.3 \%)$ & $2.4(0.7-7.6)$ & $\mathrm{p}=0.012^{\prime \prime}$ \\
\hline \multicolumn{5}{|l|}{ Laryngeal Dysfunction } \\
\hline No & $179(92.3 \%)$ & $34(19.0 \%)$ & 1.0 (referent) & \\
\hline Yes & $15(7.7 \%)$ & $2(13.3 \%)$ & $0.7(0.2-2.6)$ & $\mathrm{P}=0.574$ \\
\hline \multicolumn{5}{|l|}{ Tumor Site } \\
\hline Glottic/subglottic & $102(52.6 \%)$ & $14(13.7 \%)$ & 1.0 (referent) & \\
\hline Supraglottic & $56(28.9 \%)$ & $11(19.6 \%)$ & $1.4(0.7-2.9)$ & \\
\hline Hypopharynx & $21(10.8 \%)$ & $7(33.3 \%)$ & $2.4(1.1-5.3)$ & \\
\hline Other & $15(7.7 \%)$ & $4(26.7 \%)$ & $1.9(0.7-5.1)$ & $\mathrm{p}=0.177^{/ /}$ \\
\hline \multicolumn{5}{|l|}{ Pathologic Diagnosis } \\
\hline SCCA & $177(91.2 \%)$ & $34(19.2 \%)$ & 1.0 (referent) & \\
\hline Other & $17(8.8 \%)$ & $2(11.8 \%)$ & $0.6(0.2-2.3)$ & $\mathrm{p}=0.428$ \\
\hline \multicolumn{5}{|l|}{ Type of Surgery } \\
\hline $\mathrm{TL}$ & $147(75.8 \%)$ & $24(16.3 \%)$ & 1.0 (referent) & \\
\hline $\mathrm{TL}+$ partial pharyngectomy & $26(13.4 \%)$ & $5(19.2 \%)$ & $1.2(0.5-2.8)$ & \\
\hline TLP & $21(10.8 \%)$ & $7(33.3 \%)$ & $2.0(1.0-4.14)$ & $\mathrm{p}=0.212^{\prime \prime}$ \\
\hline \multicolumn{5}{|l|}{ Type of Reconstruction } \\
\hline None & $143(73.7 \%)$ & $23(16.1 \%)$ & 1.0 (referent) & \\
\hline Patch & $28(14.4 \%)$ & $6(21.4 \%)$ & $1.3(0.6-3.0)$ & \\
\hline Circumferential & $23(11.9 \%)$ & $7(30.4 \%)$ & $1.9(0.9-3.9)$ & $p=0.269$ \\
\hline \multicolumn{5}{|l|}{ Neck Dissection } \\
\hline No & $73(37.6 \%)$ & $12(16.4 \%)$ & 1.0 (referent) & \\
\hline Yes & $121(62.4 \%)$ & $24(19.8 \%)$ & $1.2(0.6-2.3)$ & $\mathrm{p}=0.553$ \\
\hline \multicolumn{5}{|l|}{ Thyroidectomy } \\
\hline No & $88(45.4 \%)$ & $20(22.7 \%)$ & 1.0 (referent) & \\
\hline Yes & $106(54.6 \%)$ & $16(15.1 \%)$ & $0.7(0.4-1.2)$ & $\mathrm{p}=0.174^{\prime \prime}$ \\
\hline
\end{tabular}




\begin{tabular}{|c|c|c|c|c|}
\hline & Total No. (\%) & Enlarged TEP No. (\%) & Crude RR $(95 \%$ CI) & LR-Test \\
\hline \multicolumn{5}{|l|}{ Subglottic/Tracheal Resection } \\
\hline No & $87(44.9 \%)$ & $17(19.5 \%)$ & 1.0 (referent) & \\
\hline Yes & $107(55.2 \%)$ & $19(17.8 \%)$ & $0.9(0.5-1.6)$ & $\mathrm{p}=0.751$ \\
\hline \multicolumn{5}{|l|}{ Timing TEP } \\
\hline Primary & $119(61.3 \%)$ & $18(15.1 \%)$ & 1.0 (referent) & \\
\hline Secondary & $75(38.7 \%)$ & $18(24.0 \%)$ & $1.6(0.9-2.9)$ & $\mathrm{p}=0.125^{\prime \prime}$ \\
\hline \multicolumn{5}{|l|}{ Type of TEP } \\
\hline OR & $168(86.6 \%)$ & $33(19.6 \%)$ & 1.0 (referent) & \\
\hline TNE-TEP (Clinic) & $26(13.4 \%)$ & $3(11.5 \%)$ & $0.6(0.2-1.8)$ & $\mathrm{p}=0.299$ \\
\hline \multicolumn{5}{|l|}{ Radiotherapy } \\
\hline None & $17(8.8 \%)$ & $0(0.0 \%)$ & -- & \\
\hline Preoperative & $114(58.8 \%)$ & $19(16.7 \%)$ & -- & \\
\hline Postoperative & $63(32.5 \%)$ & $17(27.0 \%)$ & -- & $\mathrm{p}=0.029^{*}$ \\
\hline \multicolumn{5}{|l|}{ Radiation prior to TEP } \\
\hline No & $64(33.0 \%)$ & $10(15.6 \%)$ & -- & \\
\hline Yes & $130(67.0 \%)$ & $26(20.0 \%)$ & $1.4(0.6-3.0)$ & $\mathrm{p}=0.456$ \\
\hline \multicolumn{5}{|l|}{ Radiation Fields } \\
\hline None & $17(8.8 \%)$ & $0(0.0 \%)$ & -- & \\
\hline Narrow-field & $43(22.2 \%)$ & $6(14.0 \%)$ & -- & \\
\hline Conventional 3D conformal & $91(46.9 \%)$ & $22(24.2 \%)$ & -- & \\
\hline IMRT & $34(17.5 \%)$ & $7(20.6 \%)$ & -- & \\
\hline Missing & $9(4.6 \%)$ & & -- & $\mathrm{p}=0.147^{*}$ \\
\hline \multicolumn{5}{|l|}{$>1$ Course Radiation } \\
\hline No & $183(94.3 \%)$ & $34(18.6 \%)$ & 1.0 (referent) & \\
\hline Yes & $11(5.7 \%)$ & $2(18.2 \%)$ & $1.0(0.3-3.6)$ & $\mathrm{p}=0.973$ \\
\hline \multicolumn{5}{|l|}{ Radiation Dose } \\
\hline Mean (sd) & $57.2(21.0)$ & $64.5(4.8)$ & $1.2(1.0-1.4)^{\dagger}$ & $\mathrm{p}=0.012^{\prime \prime}$ \\
\hline Missing & $31(16.0 \%)$ & $6(19.4 \%)$ & -- & \\
\hline \multicolumn{5}{|l|}{ Chemotherapy } \\
\hline None & $107(55.2 \%)$ & $15(14.0 \%)$ & 1.0 (referent) & \\
\hline Preoperative & $31(16.0 \%)$ & $4(12.9 \%)$ & $0.9(0.3-2.6)$ & \\
\hline Postoperative & $56(28.9 \%)$ & $17(30.4 \%)$ & $2.2(1.2-4.0)$ & $\mathrm{p}=0.033^{\prime \prime}$ \\
\hline \multicolumn{5}{|c|}{ Locoregional Recurrence or Distant Metastasis after TL } \\
\hline No & $149(76.8 \%)$ & $20(13.4 \%)$ & 1.0 (referent) & \\
\hline Yes & $45(23.2 \%)$ & $16(35.6 \%)$ & $2.6(1.5-4.7)$ & $\mathrm{p}=0.002^{\prime \prime}$ \\
\hline \multicolumn{5}{|l|}{ Largest Prosthetic Diameter } \\
\hline 16-17 French & $17(8.8 \%)$ & $3(17.7 \%)$ & 1.0 (referent) & \\
\hline 20 French & $133(68.6 \%)$ & $24(18.1 \%)$ & $1.0(0.3-3.0)$ & \\
\hline 22.5 French & $42(21.7 \%)$ & $7(16.7 \%)$ & $0.9(0.3-3.2)$ & $\mathrm{p}=0.979$ \\
\hline N/A- enlarged initial fit & $2(1.0 \%)$ & $2(100.0 \%)$ & -- & \\
\hline
\end{tabular}




\begin{tabular}{lrrrr}
\hline & Total No. $(\%)$ & Enlarged TEP No. $(\%)$ & Crude RR $(\mathbf{9 5 \%}$ CI) & LR-Test \\
\hline Insertion Method & & & & \\
$\quad$ Retrograde with stick & $170(87.6 \%)$ & $31(18.2 \%)$ & 1.0 (referent) & \\
$\quad$ Retrograde with expandable tube & $22(11.3 \%)$ & $3(13.6 \%)$ & $0.7(0.2-2.2)$ & $\mathrm{p}=0.584$ \\
Days dilation to 20-French & & $2(100.0 \%)$ & & $\mathrm{p}=0.574$ \\
$\quad$ Mean days post TEP (sd) & $70.3(116.8)$ & $57.4(81.2)$ & $1.0(1.0-1.0)$ & \\
Length of Follow-up & & & & \\
$\quad$ Mean mos. post TEP (sd) & $24.0(16.9)$ & $30.6(19.7)$ & $1.3(1.1-1.5)^{\S}$ & $\mathrm{p}=0.012^{\prime \prime}$ \\
\hline
\end{tabular}

Abbreviations: RR, relative risk; LR, likelihood ratio chi-square test; SCCA, squamous cell carcinoma; TL, total laryngectomy; TLP, total laryngopharyngectomy; TEP, tracheoesophageal puncture; 3D, three-dimensional; IMRT, intensity modulated radiation therapy; sd, standard deviation

*

Pearson chi-square

${ }^{\dagger}$ RR for every 5-Gy increase in total radiation dose

If enlarged, largest diameter and most common insertion method prior to date of enlargement

$\S_{\text {RR for every } 12 \text { month increase in length of follow-up }}$

"Candidate variable for multiple logistic regression model 
Table 3

Final Adjusted Multiple Logistic Regression Model for Enlarged TEP

\begin{tabular}{|c|c|c|c|c|}
\hline & Enlarged No. (\%) & Not Enlarged No. (\%) & Crude OR (95\% CI) & Adjusted $^{*}$ OR $(95 \% \mathrm{CI})$ \\
\hline \multicolumn{5}{|l|}{$\mathrm{N}$ classification } \\
\hline No & $3(8.3 \%)$ & $38(24.1 \%)$ & 1.0 (referent) & 1.0 (referent) \\
\hline N1 & $3(8.3 \%)$ & $9(5.7 \%)$ & $4.2(0.7-24.5)$ & $1.8(0.3-13.2)$ \\
\hline $\mathrm{N} 2$ or $\mathrm{N} 3$ & $12(33.3 \%)$ & $22(13.9 \%)$ & $6.9(1.8-27.2)$ & $4.3(1.0-19.1)$ \\
\hline $\mathrm{NX}$ or missing & $0(0.0 \%)$ & $3(1.9 \%)$ & -- & -- \\
\hline Recurrent & $18(50.0 \%)$ & $86(54.4 \%)$ & $2.7(0.7-9.5)$ & $1.7(0.4-6.7)$ \\
\hline \multicolumn{5}{|l|}{ Timing TEP } \\
\hline Primary & $18(50.0 \%)$ & $101(63.9 \%)$ & 1.0 (referent) & 1.0 (referent) \\
\hline Secondary & $18(50.0 \%)$ & $57(36.1 \%)$ & $1.8(0.9-3.7)$ & $2.5(1.0-5.9)$ \\
\hline \multicolumn{5}{|l|}{ Recurrent cancer $^{\dagger}$} \\
\hline No & $20(55.6 \%)$ & $129(81.7 \%)$ & 1.0 (referent) & 1.0 (referent) \\
\hline Yes & $16(44.4 \%)$ & $29(18.4 \%)$ & $3.6(1.6-7.7)$ & $6.2(2.3-16.4)$ \\
\hline \multicolumn{5}{|l|}{ Postoperative stricture } \\
\hline No & $25(69.4 \%)$ & $137(86.7 \%)$ & 1.0 (referent) & 1.0 (referent) \\
\hline Yes & $11(30.6 \%)$ & $21(13.3 \%)$ & $2.9(1.2-6.7)$ & $3.2(1.2-8.6)$ \\
\hline Length Follow-up (Mos.) & & & $1.0(1.0-1.1)$ & $1.0(1.0-1.1)$ \\
\hline
\end{tabular}

Abbreviations: OR, odds ratio; CI, confidence interval; TEP, tracheoesophageal puncture

* Adjusted for all variables in multiple logistic model including N-stage, timing of TEP, recurrent cancer after laryngectomy, postoperative stricture, and length of follow-up.

${ }^{\dagger}$ Locoregional recurrence or distant metastasis diagnosed after total laryngectomy 